\title{
Estimation of the Bid-Ask Prices for the European Discrete Geometric Average and Arithmetic Average Asian Options
}

\author{
Xiankang Luo and Tao Chen (iD \\ Faculty of Science Yibin University, Yibin 644000, China \\ Correspondence should be addressed to Tao Chen; shuxue_e@163.com
}

Received 21 March 2021; Accepted 31 May 2021; Published 8 June 2021

Academic Editor: Tien Van Do

Copyright (c) 2021 Xiankang Luo and Tao Chen. This is an open access article distributed under the Creative Commons Attribution License, which permits unrestricted use, distribution, and reproduction in any medium, provided the original work is properly cited.

Conic finance is a new and exciting development in quantitative finance, which is widely applied to several topics in finance. The theory of conic finance extends the law of one price to the law of two prices, which yields closed forms for bid-ask prices of European options. In this paper, within the framework of conic finance, we derive effective, explicit, approximate formulas to estimate the bid-ask prices for the European discrete geometric average and arithmetic average Asian options. Finally, we give two examples to demonstrate and validate that the approximate closed-form solutions are efficient and accurate.

\section{Introduction}

In the OTC markets, the Asian option is one of the most popular exotic options. Asian options are options whose payoffs depend on some average price of the underlying asset over some prescribed period; Asian options are also called average price options. There are two main advantages of Asian options: one for reducing the risk of market manipulation of the underlying instrument at maturity and the other one for reducing the volatility inherent in the option. Because of the path-dependent characteristic, Asian options have a lower volatility and a lower cost than American options [1]. Therefore, Asian options have attracted much attention, and their volume has grown rapidly in the OTC markets.

In the Black-Scholes-Merton framework, many scholars have sufficient research on exotic options. They develop many methods such as chaos theory, fractal Brownian motion, time-changed Brownian, stochastic volatility, and models based on Lévy processes to incorporate two empirical features: the asymmetric leptokurtic features and the volatility smile $[2,3]$. In a parallel development, many mathematical methods are used to solve the value of options, such as the numerical method (PDE numerical calculation, binomial lattice, Monte Carlo simulation, and Fourier transform), partial differential equation method, and deep learning method [4-6]. These methods are well known and can be found in many option pricing textbooks. However, there are many uncertain factors in the real financial market, which profoundly affect the option pricing, so there is no effective method to solve the option value. As we all know, classical option pricing theories are usually built on the law of one price or the risk-neutral price which assumes implicitly that market participants can buy or sell any amount of assets with the market taking the opposite position. However, empirical studies showed that even in highly liquid financial markets, we observe two prices, namely, the price at which the market is willing to buy (bid) and a price at which the market is willing to sell (ask). When liquidity is insufficient, it can lead to a higher difference between the bid price and ask price, and we can no longer rely on the law of one price.

There are a variety of theoretical approaches that try to model bid-ask spreads (see Madan and Cherny [7] for general reviews). A different approach was taken by Madan and Cherny [7] which is based on the theory of conic finance, originating from the framework of acceptability indices presented in Cherny and Madan [8]. In the conic finance framework, the price depends on the direction of trade, one for buying from the market at the ask price and another for 
selling to the market at the bid price. Conic finance is a new and exciting development in quantitative finance, which is widely applied to several topics in finance. One of the applications is option pricing with liquidity. In [7], Madan and Cherny established the conic finance theory by using the acceptability indices' theory and the nonlinear expectation theory and derived the general closed-form formulas for bid and ask prices for European options. In 2010, Madan [9] studied the discrete-time constant liquidity model. Leippold and Schärer [10] first proved that multiperiod bid-ask prices with stochastic liquidity are time-consistent and dynamically translation-invariant nonlinear expectations. The results extend the model of Madan [9] to develop a discrete-time option pricing model with stochastic liquidity and open ample ground for future research. Furthermore, Leippold and Schärer [11] developed the conic finance framework for optimal execution strategies with stochastic liquidity. In [12-15], the scholars made an empirical analysis of the option pricing model under the conic finance framework. The empirical results showed the huge advantage of conic finance theory in modeling liquidity.

At the same time, many research topics including conic CVA and DVA, conic portfolio theory, conic hedging of financial and insurance risks, and conic trading are presented in the recently published book by Madan and Schoutens [16]. Recently, the researchers derived the explicit formulas for the bid and ask prices of European vanilla options [15, 16], interest rate options [17], and continuous geometric average Asian options [18], respectively, by using the conic finance theory. To the best of our knowledge, there is no literature research to estimate the bid-ask prices of the discrete Asian options. In this paper, motivated by the conic finance theory, we derive an effective, explicit, approximate formula to calculate the bid and ask prices for the discrete Asian options including discrete geometric average and arithmetic average Asian options.

The content of this paper is organized as follows. In Section 2, we review the conic finance theory. Section 3 is devoted to derive an effective, explicit, approximate formula to calculate the bid and ask prices for the discrete Asian options. In Section 4, we present two numerical examples which show that the approximate closed forms are efficient and accurate. Section 5 concludes the paper.

\section{Conic Finance Theory}

Conic finance is a new and exciting development in quantitative finance, which extends the law of one price to the law of two prices. The conic finance theory originates from the framework of acceptability developed by Cherny and Madan [8]. In the conic finance framework, there are two prices for financial assets in the market. If a nonnegative stochastic cash flow $X$ is acceptable at liquidity level $\gamma$, the market maker buys at the ask price $a_{\gamma}(X)$ and sells at the bid price $b_{\gamma}(X)$. The difference of bid and ask prices gives rise to the bid-ask spread which can measure market liquidity.

The foundation of the conic finance theory is an underlying concept of acceptable risk in the economy. The theory of acceptability indices is developed in [8], and the index of acceptability is a new measure for performance evaluation which is a function $\alpha: L^{\infty} \longrightarrow[0, \infty)$, where $L^{\infty}:=L^{\infty}(\Omega, \mathscr{F}, \mathbb{P})$ is the space of all essentially bounded. For the acceptability index $\alpha(X)$ and for each liquidity level $\gamma>0$, there is a family of sets of probability measures $\left(D_{\gamma}\right)_{\gamma \geq 0}$ equivalent to the initial probability measure of $\mathbb{P}$. A risk $X$ is acceptable at level $\gamma$ if and only if $\mathbb{E}^{\mathbb{Q}}[X] \geq 0$, for all $\mathbb{Q} \in \mathscr{D}_{\gamma}$. That is to say, the acceptability index $\alpha(\widetilde{X})$ will satisfy the following operational acceptability level:

$$
\alpha(X)=\sup \left\{\gamma \geq 0: \mathbb{E}^{\mathbb{Q}}[X] \geq 0, \quad \text { for any } \mathbb{Q} \in \mathscr{D}_{\gamma}\right\} .
$$

Suppose the trader, due to competition, will sell a net cash flow $X$; it charges a minimal price $a$. The residual cash flow $a-X$ ought to be $\alpha$-acceptable at level $\gamma$. For $a-X$ to be acceptable at level $\gamma$, the price $a$ must exceed $\mathbb{E}^{\mathbb{Q}}[X] \geq 0$ for any $\mathbb{Q} \in \mathscr{D}_{\gamma}$, so the ask price is derived as follows:

$$
\begin{aligned}
a_{\gamma}(X) & =\inf \{a: \alpha(a-X) \geq \gamma\} \\
& =\inf \left\{a: \mathbb{E}^{\mathbb{Q}}[a-X] \geq \gamma \text { for any } \mathbb{Q} \in \mathscr{D}_{\gamma}\right\} \\
& =\sup _{\mathbb{Q} \in D_{\gamma}} \mathbb{E}^{\mathbb{Q}}[X] .
\end{aligned}
$$

For the buy-side, when the market maker buys a net cash flow $X$, again driven by the competition, he charges a maximum price of $b$. The residual cash flow $X-b$ must be $\alpha$-acceptable at level $\gamma$. Hence, the maximal price is

$$
b_{\gamma}(X)=\inf _{\mathbb{Q} \in D_{\gamma}} \mathbb{E}^{\mathbb{Q}}[X] .
$$

As proposed by Cherny and Madan [8], Choquet expectation (see [19]) based on the concave distortion function can be used to formulate an operational index of acceptability. The distorted expectation of a risk $X$ with distribution function $F_{X}(x)$ relative to the concave distortion function $\left(\psi^{\gamma}\right)_{\gamma \geq 0}$ is defined as

$$
\mathbb{E}^{\psi^{\gamma}}[X]=\int_{-\infty}^{\infty} x d \psi^{\gamma}\left(F_{X}(x)\right)
$$

and the acceptability index $\alpha(X)$ is characterized as

$$
\alpha(X)=\sup \left\{\gamma \geq 0: \int_{-\infty}^{\infty} x d \psi^{\gamma}\left(F_{X}(x)\right)\right\},
$$

where $X$ is a nonnegative stochastic variable, $F_{X}(x)$ is the distribution function of $X$, and $\left(\psi^{\gamma}\right)_{\gamma \geq 0}$ is a pointwise increasing family of concave distortion functions. Because the distortion function is an important bridge between Choquet expectation (nonlinear expectation) and linear expectation, Cherny and Madan [8] concluded a series of distortion functions $\left(\psi^{\gamma}\right)_{\gamma \geq 0}$, such as MINVAR, MAXVAR, MAXMINVAR, and MINMAXVAR. In this paper, we need to use the Choquet expectation on Wang transform, so we first give related definitions as follows.

Definition 1 (distortion function). A function $\psi:[0,1] \longrightarrow$ $[0,1]$ is a distortion function if and only if it is monotone, $\psi(0)=0$ and $\psi(1)=1$. 
Definition 2 (Wang transform; see $[20,21]$ ). Let $\Phi$ denote the standard normal cumulative distribution function, and let $\gamma$ be a nonnegative constant. Then, a distortion function $\psi^{\gamma}:[0,1] \longrightarrow[0,1]$ defined by

$$
\psi^{\gamma}(u)=\Phi\left(\Phi^{-1}(u)+\gamma\right)
$$

is called the Wang transform.

By using the distortion expectation (4) and a family of concave distortion functions $\left(\psi^{\gamma}\right)_{\gamma \geq 0}$, the ask price is derived as follows:

$$
\begin{aligned}
\alpha(a-X) \geq \gamma \Rightarrow & \int_{-\infty}^{\infty} x d \psi^{\gamma}\left(F_{a-X}(x)\right) \geq 0 \Rightarrow a \\
& +\int_{-\infty}^{\infty} x d \psi^{\gamma}\left(F_{-X}(x)\right) \geq 0 .
\end{aligned}
$$

so that the minimum price of $a$ leads to the ask price:

$$
a_{\gamma}(X)=-\int_{-\infty}^{\infty} x d \psi^{\gamma}\left(F_{-X}(x)\right) \text {. }
$$

Similarly, the maximum price of $b$ leads to the bid price:

$$
\begin{aligned}
\alpha(X-b) \geq \gamma \Rightarrow & \int_{-\infty}^{\infty} x d \psi^{\gamma}\left(F_{X-b}(x)\right) \geq 0 \Rightarrow-b \\
& +\int_{-\infty}^{\infty} x d \psi^{\gamma}\left(F_{X}(x)\right) \geq 0 .
\end{aligned}
$$

We obtain

$$
b_{\gamma}(X)=\int_{-\infty}^{\infty} x d \psi^{\gamma}\left(F_{X}(x)\right)
$$

In order to present the relationship between the distorted expectation and classical integral, Madan and Cherny [7] led to the following definition of bid and ask prices.

Definition 3. (single-period bid-ask prices; see [7]). Let $\left(\psi^{\gamma}\right)_{\gamma \geq 0}$ be a pointwise increasing family of concave distortion functions and $\gamma$ be the market liquidity level. Then, the bid price of a discounted cash flow $X \in L^{\infty}$ is given by

$$
\begin{aligned}
b_{\gamma}(X)= & \mathbb{E}^{\psi^{\gamma}}[X] \\
= & (C) \int X \mathrm{~d} \psi^{\gamma} \circ \mathbb{P} \\
= & -\int_{-\infty}^{0} \psi^{\gamma}(\mathbb{P}(X \leq x)) \mathrm{d} x \\
& +\int_{0}^{\infty}\left(1-\psi^{\gamma}(\mathbb{P}(X \leq x))\right) \mathrm{d} x,
\end{aligned}
$$

and its ask price is

$$
\begin{aligned}
a_{\gamma}(X)= & -\mathbb{E}^{\psi^{\gamma}}[-X] \\
= & \int_{-\infty}^{0}\left(\psi^{\gamma}(\mathbb{P}(X>x))-1\right) \mathrm{d} x \\
& +\int_{0}^{\infty} \psi^{\gamma}(\mathbb{P}(X>x)) \mathrm{d} x .
\end{aligned}
$$

In particular, the Choquet expectation, introduced by Choquet in [19], is a nonlinear expectation when the market liquidity level $\gamma=0$, the distortion expectation reduces to a linear expectation on the reference pricing measure $\mathbb{P}$, and bid-ask prices (8)-(10) are equivalent. In addition, the concavity of the distortion ensures that the ask price is greater than the bid price, i.e.,

$$
b_{\gamma}(X) \leq \mathbb{E}[X] \leq a_{\gamma}(X),
$$

where $\mathbb{E}[X]$ is a linear expectation on the reference pricing measure $\mathbb{P}$, which means that distortion is not applied at all.

\section{The Bid-Ask Prices of Discrete Average Asian Options}

In this section, we first introduce the pricing of discrete average Asian options under the law of one price. Second, within the framework of conic finance, we derive effective, explicit, approximate formulas to calculate the bid and ask prices for the discrete Asian options including discrete geometric average and arithmetic average Asian options.

\subsection{Discrete Average Asian Option under the Law of One Price.} In this section, we start with a brief description of the discrete geometric and arithmetic Asian option under the law of one price.

Under a probability space $(\Omega, \mathscr{F}, \mathbb{P})$, the reference probability measure $\mathbb{P}$ is assumed to be a risk-neutral measure. It is assumed that the underlying asset follows the geometric Brownian motion, with a stochastic differential equation having the following expression:

$$
\mathrm{d} S_{t}=r S_{t} \mathrm{~d} t+\sigma S_{t} \mathrm{~d} W_{t}, \quad 0 \leq t \leq T, S(0)=S_{0},
$$

where $r$ is the risk-free rate and $\sigma$ is volatility. And these parameters are usually assumed to be constant. The solution of equation (14) can be calculated to the following result:

$$
S_{T}=S_{t} \exp \left(\left(r-\frac{\sigma^{2}}{2}\right) \tau+\sigma Z_{\tau}\right),
$$

where $\tau=T-t$ is the time to maturity and $Z_{\tau}=W_{T}-W_{t}$ is the Brownian motion.

Suppose that $n$ prices are taken from equation (15) with observation frequency $h$, i.e.,

$$
\begin{aligned}
a_{i} & =S(\tau-(n-i) h) \\
& =S_{0} \exp \left(\left(r-\frac{\sigma^{2}}{2}\right)(\tau-(n-i) h)+\sigma Z(\tau-(n-i) h)\right)
\end{aligned}
$$

where $i=1,2,3, \cdots, n$, and $\tau=T-t$ is the time to maturity. We can see that the averaging period starts with the first observation at $T=\tau-(n-1) h$ and stops at the last observation $i=n$ at $T=\tau$. The averaging time period is thus from $\tau-(n-1) h$ to $\tau$ or $(n-1) h$. 
The standard geometric average (GA) of $n$ positive prices $a_{1}, a_{2}, \cdots, a_{n}$ is defined as

$$
\mathrm{GA}(n)=\left(\prod_{i=1}^{n} a_{i}\right)^{1 / n}
$$

and the arithmetic average (AA) of $n$ positive prices $a_{1}, a_{2}, \ldots, a_{n}$ is defined as

$$
\mathrm{AA}(n)=\frac{1}{n} \sum_{i=1}^{n} a_{i}, \quad i=1,2,3, \ldots, n,
$$

where $n$ is the number of observations and $a_{i}$ is the $i$ th observation.

Let $V\left(S_{t}, t\right)$ be the option price with the underlying price $S_{t}$, the time to maturity $T$, and the strike price $K$. Then, the price of the discrete geometric Asian call option under the risk-neutral measure $\mathbb{P}$, at time $t$, may be represented as

$$
V\left(S_{t}, t\right)=e^{-r(T-t)} \mathbb{E}\left[(\mathrm{GA}(n)-K)^{+}\right],
$$

and the price of the discrete arithmetic Asian put option is given by

$$
V\left(S_{t}, t\right)=e^{-r(T-t)} \mathbb{E}\left[K-(\mathrm{AA}(n))^{+}\right],
$$

where $\mathrm{GA}(n)$ is the discrete geometric average and $\mathrm{AA}(n)$ is the arithmetic average of the underlying asset prices during the time to maturity $T$.

In order to price the discrete geometric and arithmetic Asian option, we have to know the distribution of the geometric average $\mathrm{GA}(n)$ and arithmetic average $\mathrm{AA}(n)$. In both continuous case and discrete case of the geometric Asian option, the variable GA $(n)$ is log-normally distributed so that its expectation and variance values may be calculated explicitly. Therefore, Kemma and Vorst [22], Turnbull and Wakeman, [23], and Zhang [1] obtained similar B-S pricing formulas of geometric average Asian options by different approximation methods.

Asian option trading in the OTC markets is usually based on the arithmetic average prices of the underlying assets. However, even if all price observations follow a lognormal process, the arithmetic average is not a log-normal process. It is difficult to solve the closed-form solutions for arithmetic average Asian options. A representative method was proposed in Zhang [1]. Zhang [1] tried to express the difference in terms of the distribution of the given observations using the Taylor series expansion and then obtained an approximation formula for arithmetic means in terms of their corresponding geometric means. The following lemmas come from Zhang [1], which give the distribution of $\mathrm{GA}(n)$ and $\mathrm{AA}(n)$.

Lemma 1 (see Zhang [1]). Let the averaging numbers be defined by equation (16); then, the standard geometric average $G A(n)$ is a log-normal distribution, i.e.,

$$
\operatorname{logGA}(n) \sim N\left(\left(r-\frac{1}{2} \sigma^{2}\right) T_{\mu, n-j}^{s a}+\ln B_{j}^{s a}+\ln S_{t}, \sigma^{2} T_{n-j}^{s a}\right),
$$

where

$$
\begin{aligned}
B_{0}^{s a} & =1, B_{j}^{s a}=\left(\prod_{i=1}^{j} \frac{S(\tau-(n-i) h)}{S_{0}}\right)^{1 / n}, \quad 1 \leq j \leq n, \\
T_{\mu, n-j}^{s a} & =\frac{n-j}{n}\left(\tau-\frac{(n-j-1) h}{2}\right), \\
T_{n-j}^{s a} & =\tau\left(\frac{n-j}{2}\right)^{2}-\frac{(n-j)(n-j-1)(4 n-4 j+1) h}{6 n^{2}},
\end{aligned}
$$

and $n$ is the number of observations specified in the contract, $h$ is the observation frequency or the time interval between two consecutive observations, $j$ is the number of observations already passed, $B_{j}$ is the geometric average of the gross returns of those observations that have already passed, $\tau$ is the time to maturity of the option, and the two functions $T_{\mu, n-j}$ and $T_{n-j}$ may be interpreted as the effective mean and volatility time functions, respectively, because they largely determine the effective mean and variance of the geometric average.

Lemma 2 (see Zhang [1]). The arithmetic average of $n$ price observations, each following the Brownian motion specified in (16), can be approximated with the corresponding geometric mean:

$$
\mathrm{AA}(a) \cong \kappa \mathrm{GA}(a)
$$

where

$$
\begin{aligned}
\kappa & =1+\widetilde{\mathbb{E}}\left(v+v^{2}\right) \\
& =1+\frac{1}{2} \widetilde{\mathbb{E}}[V(\ln a)]+\frac{1}{4}(\operatorname{Var}[V(\ln a)]+\widetilde{\mathbb{E}}[V(\ln a)])^{2}, \\
\widetilde{\mathbb{E}}[V(\ln a)] & =\frac{\left(n^{2}-1\right) h}{6}\left(\frac{1}{2}\left(r-\frac{1}{2} \sigma^{2}\right)^{2} h+\frac{1}{n} \sigma^{2}\right), \\
\operatorname{Var}[V(\ln a)] & =\frac{\left(n^{2}-1\right)\left(3 n^{2}-2\right)}{15 n^{3}}\left(r-\frac{1}{2} \sigma^{2}\right)^{2} \sigma^{2} h^{3} .
\end{aligned}
$$


According to Lemmas 1 and 2, we can easily derive the closed-form solution of the discrete average Asian option under the law of one price. These pricing formulas are well known and can be found in many option pricing textbooks.

3.2. The Bid-Ask Prices of Discrete Geometric Average Asian Options. In this section, within the framework of conic finance, we first derive the explicit approximate formulas for the bid-ask prices of the discrete geometric average Asian options. Furthermore, by using Lemma 2 and a similar proof method of Theorem 1, we can readily derive explicit approximate formulas for the bid-ask price of the discrete geometric average Asian options. We will give the explicit approximate formulas in the following theorems.

Theorem 1. Assume that the distortion function $\psi^{\gamma}(u)$ is the Wang transform; then, the bid-ask prices of the European discrete geometric average Asian call option at time t are given by

$$
\begin{aligned}
& b_{\gamma}(C) \cong S_{t} A_{j}^{b} \Phi\left(d_{n-j}^{b}+\sigma \sqrt{T_{n-j}^{s a}}\right)-e^{-r \tau} K \Phi\left(d_{n-j}^{b}\right), \\
& a_{\gamma}(C) \cong S_{t} A_{j}^{a} \Phi\left(d_{n-j}^{a}+\sigma \sqrt{T_{n-j}^{s a}}\right)-e^{-r \tau} K \Phi\left(d_{n-j}^{a}\right),
\end{aligned}
$$

and the bid-ask prices of the European discrete geometric average Asian put option at time t are given by

$$
\begin{aligned}
& b_{\gamma}(P) \cong e^{-r \tau} K \Phi\left(-d_{n-j}^{a}\right)-S_{t} A_{j}^{a} \Phi\left(-d_{n-j}^{a}-\sigma \sqrt{T_{n-j}^{s a}}\right), \\
& a_{\gamma}(P) \cong e^{-r \tau} K \Phi\left(-d_{n-j}^{b}\right)-S_{t} A_{j}^{b} \Phi\left(-d_{n-j}^{b}-\sigma \sqrt{T_{n-j}^{s a}}\right),
\end{aligned}
$$

where $\tau=T-t$ is maturity,

$$
\begin{aligned}
d_{n-j}^{b} & =\frac{\ln \left(B_{j}^{s a} S_{t} / K\right)+\left(r-1 / 2 \sigma^{2}\right) T_{\mu, n-j}^{s a}}{\sigma \sqrt{T_{n-j}^{s a}}}-\gamma, \\
d_{n-j}^{a} & =\frac{\ln \left(B_{j}^{s a} S_{t} / K\right)+\left(r-1 / 2 \sigma^{2}\right) T_{\mu, n-j}^{s a}}{\sigma \sqrt{T_{n-j}^{s a}}}+\gamma, \\
A_{j}^{b} & =B_{j}^{s a} \exp \left(-r\left(\tau-T_{\mu, n-j}^{s a}\right)-\frac{\sigma^{2}}{2}\left(T_{\mu, n-j}^{s a}-T_{n-j}^{s a}\right)-\gamma \sigma \sqrt{T_{n-j}^{s a}}\right), \\
A_{j}^{a} & =B_{j}^{s a} \exp \left(-r\left(\tau-T_{\mu, n-j}^{s a}\right)-\frac{\sigma^{2}}{2}\left(T_{\mu, n-j}^{s a}-T_{n-j}^{s a}\right)+\gamma \sigma \sqrt{T_{n-j}^{s a}}\right) .
\end{aligned}
$$

$B_{j}^{s a}, T_{n-j}^{s a}$, and $T_{\mu, n-j}^{s a}$ are the same as in Lemma 1. In particular, for $\gamma=0$, the bid-ask prices are equivalent, and the bid-ask prices of the European discrete geometric average Asian option reduce to the B-S-type formulas in [1].

Proof. Consider the discrete geometric average GA $(n)$ defined by equation (17). And Lemma 1 shows that random variable $\mathrm{GA}(n)$ has a log-normal distribution under riskneutral measure $\mathbb{P}$, which means that

$$
F_{\mathrm{GA}}(x)=\Phi\left(\frac{\ln \left(x /\left(B_{j}^{s a} S_{t}\right)\right)-\left(r-1 / 2 \sigma^{2}\right) T_{\mu, n-j}^{s a}}{\sigma \sqrt{T_{n-j}^{s a}}}\right),
$$

where $\Phi(\cdot)$ is the standard normal cumulative distribution function. If we apply Wang transform (6) to the distribution function $F_{\mathrm{GA}}$, we will get the following representation:

$$
\psi^{\gamma}\left(F_{\mathrm{GA}}(x)\right)=\Phi\left(\frac{\ln \left(x /\left(B_{j}^{s a} S_{t}\right)\right)-\left(r-1 / 2 \sigma^{2}\right) T_{\mu, n-j}^{s a}}{\sigma \sqrt{T_{n-j}^{s a}}}+\gamma\right) .
$$

Let the payoff of the European discrete geometric average Asian call option be $C_{T}=(\mathrm{GA}(n)-K)^{+}$; we can derive the bid price for a European discrete geometric average Asian call option by Choquet integral:

$$
\begin{aligned}
b_{\gamma}(C) & =e^{-r \tau} \mathbb{E}^{\psi^{\gamma}}\left[C_{T}\right]=e^{-r \tau}(C) \int C_{T} d \psi^{\gamma} \circ \mathbb{P} \\
& =e^{-r \tau} \int_{0}^{\infty} x d \psi^{\gamma}\left(F_{C_{T}}(x)\right) \\
& =e^{-r \tau} \int_{K}^{\infty}(x-K) d \psi^{\gamma}\left(F_{\mathrm{GT}}(x)\right) \\
& =e^{-r \tau} \int_{K}^{\infty} x d \psi^{\gamma}\left(F_{\mathrm{GT}}(x)\right)-\int_{K}^{\infty} K d \psi^{\gamma}\left(F_{\mathrm{GT}}(x)\right) \\
& :=I_{1}-I_{2} .
\end{aligned}
$$


Neglecting the discount factor $e^{-r \tau}$ temporarily, we can calculate the integral $I_{1}$ in equation (32). It is shown that

$$
\begin{aligned}
I_{1} & =\int_{K}^{\infty} x d \psi^{\gamma}\left(F_{\mathrm{GA}}(x)\right) \\
& =\int_{K}^{\infty} x d \Phi\left(\frac{\ln \left(x /\left(B_{j}^{s a} S_{t}\right)\right)-\left(r-1 / 2 \sigma^{2}\right) T_{\mu, n-j}^{s a}}{\sigma \sqrt{T_{n-j}^{s a}}}+\gamma\right) \\
& =\exp \left(r T_{\mu, n-j}^{s a}-\frac{\sigma^{2}}{2}\left(T_{\mu, n-j}^{s a}-T_{n-j}^{s a}\right)+\ln \left(B_{j}^{s a} S_{t}\right)-\gamma \sigma \sqrt{T_{n-j}^{s a}}\right) \cdot \Phi\left(\frac{\ln \left(B_{j}^{s a} S_{t} / K\right)+\left(r-1 / 2 \sigma^{2}\right) T_{\mu, n-j}^{s a}+\sigma^{2} T_{n-j}^{s a}}{\sigma \sqrt{T_{n-j}^{s a}}}-\gamma\right) .
\end{aligned}
$$

And the second integral $I_{2}$ in equation (32) can be calculated as

$$
\begin{aligned}
I_{2} & =\int_{K}^{\infty} K d \psi^{\gamma}\left(F_{\mathrm{GT}}(x)\right) \\
& =\int_{K}^{\infty} K d \Phi\left(\frac{\ln \left(x /\left(B_{j}^{s a} S_{t}\right)\right)-\left(r-1 / 2 \sigma^{2}\right) T_{\mu, n-j}^{s a}}{\sigma \sqrt{T_{n-j}^{s a}}}+\gamma\right) \\
& =K\left(1-\Phi\left(\frac{\ln \left(K /\left(B_{j}^{s a} S_{t}\right)\right)-\left(r-1 / 2 \sigma^{2}\right) T_{\mu, n-j}^{s a}}{\sigma \sqrt{T_{n-j}^{s a}}}+\gamma\right)\right) \\
& =K \Phi\left(\frac{\ln \left(\left(B_{j}^{s a} S_{t}\right) / K\right)+\left(r-1 / 2 \sigma^{2}\right) T_{\mu, n-j}^{s a}}{\sigma \sqrt{T_{n-j}^{s a}}}\right) .
\end{aligned}
$$

Substituting equations (33) and (34) into (32) and multiplying by a discount factor $e^{-r \tau}$, we can get the following expression for the bid price:

$$
\begin{aligned}
b_{\gamma}(C)= & B_{j}^{s a} S_{t} \exp \left(-r\left(\tau-T_{\mu, n-j}^{s a}\right)-\frac{\sigma^{2}}{2}\left(T_{\mu, n-j}^{s a}-T_{n-j}^{s a}\right)-\gamma \sigma \sqrt{T_{n-j}^{s a}}\right) \cdot \Phi\left(\frac{\ln \left(B_{j}^{s a} S_{t} / K\right)+\left(r-1 / 2 \sigma^{2}\right) T_{\mu, n-j}^{s a}+\sigma^{2} T_{n-j}^{s a}}{\sigma \sqrt{T_{n-j}^{s a}}}-\gamma\right) \\
& -e^{-r \tau} K \Phi\left(\frac{\ln \left(B^{s a} S_{t} / K\right)+\left(r-1 / 2 \sigma^{2}\right) T_{\mu, n-j}^{s a}}{\sigma \sqrt{T_{n-j}^{s a}}}-\gamma\right) .
\end{aligned}
$$


Now, by using Choquet expectation in Definition 3, we can derive the ask price of the European discrete geometric average Asian call option:

$$
\begin{aligned}
a_{\gamma}(C)= & -\mathbb{E}^{\psi^{\gamma}}\left[-C_{T}\right] \\
= & -\int_{-\infty}^{0} x d \psi^{\gamma}\left(F_{-C_{T}}(x)\right) \\
= & -\int_{-\infty}^{0} x d \psi^{\gamma}\left(1-F_{\mathrm{GA}}(K-x)\right) \\
= & -\int_{0}^{\infty} x d \psi^{\gamma}\left(1-F_{\mathrm{GA}}(K+x)\right) \\
= & -\int_{K}^{\infty}(x-K) d \psi^{\gamma}\left(1-F_{\mathrm{GA}}(x)\right) \\
= & -\int_{K}^{\infty} x d \psi^{\gamma}\left(1-F_{\mathrm{GA}}(x)\right) \\
& +\int_{K}^{\infty} K d \psi^{\gamma}\left(1-F_{\mathrm{GA}}(x)\right) \\
= & \bar{I}_{1}+\bar{I}_{2} .
\end{aligned}
$$

Similar to the way in which we obtained the bid price, we get the first integral in equation (36) as follows:

$$
\begin{aligned}
\bar{I}_{1} & =-\int_{K}^{\infty} x d \psi^{\gamma}\left(1-F_{\mathrm{GA}}(x)\right) \\
& =-\int_{K}^{\infty} x \mathrm{~d} \Phi\left(\frac{\ln \left(\left(B_{j}^{s a} S_{t}\right) / x\right)+\left(r-1 / 2 \sigma^{2}\right) T_{\mu, n-j}^{s a}}{\sigma \sqrt{T_{n-j}^{s a}}}+\gamma\right) \\
& =\int_{K}^{\infty} x \mathrm{~d} \Phi\left(\frac{\ln \left(x /\left(B_{j}^{s a} S_{t}\right)\right)-\left(r-1 / 2 \sigma^{2}\right) T_{\mu, n-j}^{s a}}{\sigma \sqrt{T_{n-j}^{s a}}}-\gamma\right) \\
& =\exp \left(r T_{\mu, n-j}^{s a}-\frac{\sigma^{2}}{2}\left(T_{\mu, n-j}^{s a}-T_{n-j}^{s a}\right)+\ln \left(B_{j}^{s a} S_{t}\right)+\gamma \sigma \sqrt{T_{n-j}^{s a}}\right) \cdot \Phi\left(\frac{\ln \left(B_{j}^{s a} S_{t} / K\right)+\left(r-1 / 2 \sigma^{2}\right) T_{\mu, n-j}^{s a}+\sigma^{2} T_{n-j}^{s a}}{T_{n-j}}+\gamma\right),
\end{aligned}
$$

and the second integral can be calculated as

$$
\begin{aligned}
\bar{I}_{2} & =\int_{K}^{\infty} K \mathrm{~d} \psi^{\gamma}\left(1-F_{\mathrm{GA}}(x)\right) \\
& =\int_{K}^{\infty} K \mathrm{~d} \Phi\left(\frac{\ln \left(\left(B_{j}^{s a} S_{t}\right) / x\right)+\left(r-1 / 2 \sigma^{2}\right) T_{\mu, n-j}^{s a}}{\sigma \sqrt{T_{n-j}^{s a}}}+\gamma\right) \\
& =-\int_{K}^{\infty} K \mathrm{~d} \Phi\left(\frac{\ln \left(x /\left(B_{j}^{s a} S_{t}\right)\right)-\left(r-1 / 2 \sigma^{2}\right) T_{\mu, n-j}^{s a}}{\sigma \sqrt{T_{n-j}^{s a}}}-\gamma\right) \\
& =-K\left(1-\Phi\left(\frac{\ln \left(K /\left(B_{j}^{s a} S_{t}\right)\right)-\left(r-1 / 2 \sigma^{2}\right) T_{\mu, n-j}^{s a}}{\sigma \sqrt{T_{n-j}^{s a}}}-\gamma\right)\right) \\
& =-K \Phi\left(\frac{\ln \left(B_{j}^{s a} S_{t} / K\right)+\left(r-1 / 2 \sigma^{2}\right) T_{\mu, n-j}^{s a}}{\sigma}+\gamma\right) .
\end{aligned}
$$


By combining parts $\bar{I}_{1}$ and $\bar{I}_{2}$ and considering the continuous discount factor $e^{-r \tau}$, we have the ask price:

$$
\begin{aligned}
a_{\gamma}(C)= & B_{j}^{s a} S_{t} \exp \left(-r\left(\tau-T_{\mu, n-j}^{s a}\right)-\frac{\sigma^{2}}{2}\left(T_{\mu, n-j}^{s a}-T_{n-j}^{s a}\right)+\sigma \sqrt{T_{n-j}^{s a}}\right) \cdot \Phi\left(\frac{\ln \left(B_{j}^{s a} S_{t} / K\right)+\left(r-1 / 2 \sigma^{2}\right) T_{\mu, n-j}^{s a}+\sigma^{2} T_{n-j}^{s a}}{\sigma \sqrt{T_{n-j}^{s a}}}\right) \\
& -e^{-r \tau} K \Phi\left(\frac{\ln \left(B_{j}^{s a} S_{t} / K\right)+\left(r-1 / 2 \sigma^{2}\right) T_{\mu, n-j}^{s a}}{\sigma \sqrt{T_{n-j}^{s a}}}+\gamma\right) .
\end{aligned}
$$

In addition, let the put option payoff be $P_{T}=(K-\mathrm{GA}(n))^{+}$. Similar to the way in which we derived the bid-ask prices of the call option, we can derive the bid- ask prices of the European discrete geometric average Asian put option. We first gave the bid price of the put option as follows:

$$
\begin{aligned}
b_{\gamma}(P) & =e^{-r \tau} \mathbb{E}^{\psi^{\gamma}}\left[P_{T}\right]=e^{-r \tau} \int_{0}^{\infty} x \mathrm{~d} \psi^{\gamma}\left(F_{P_{T}}(x)\right) \\
& =e^{-r \tau} \int_{0}^{\infty} x \mathrm{~d} \psi^{\gamma}\left(1-F_{\mathrm{GA}}(K-x)\right) \\
& =-e^{-r \tau} \int_{0}^{K}(K-x) \mathrm{d} \psi^{\gamma}\left(1-F_{\mathrm{GA}}(x)\right) \\
& =e^{-r \tau} \int_{0}^{K}(K-x) \mathrm{d} \Phi\left(\frac{\ln \left(x /\left(B_{j}^{s a} S_{t}\right)\right)-\left(r-1 / 2 \sigma^{2}\right) T_{\mu, n-j}^{s a}}{\sigma}-\gamma\right) \\
& \left.=e^{-r \tau} K \Phi\left(\frac{\ln \left(K /\left(B_{j}^{s a} S_{t}\right)\right)-\left(r-1 / 2 \sigma^{2}\right) T_{\mu, n-j}^{s a}}{\sigma a}\right) \gamma\right) \\
& -B_{j}^{s a} S_{t} \exp \left(-r\left(\tau-T_{\mu, n-j}^{s a}\right)-\frac{\sigma^{2}}{2}\left(T_{\mu, n-j}^{s a}-T_{n-j}^{s a}\right)+\gamma \sigma \sqrt{T_{n-j}^{s a}}\right) \cdot \Phi\left(\frac{\ln \left(K /\left(B_{j}^{s a} S_{t}\right)\right)-\left(r-1 / 2 \sigma^{2}\right) T_{\mu, n-j}^{s a}-\sigma^{2} T_{n-j}^{s a}}{\sigma}-\gamma\right) .
\end{aligned}
$$

Furthermore, we can also get the formula of the ask price of the European discrete geometric average Asian put option: 


$$
\begin{aligned}
& a_{\gamma}(P)=-e^{-r \tau} \mathbb{E}^{\psi^{\gamma}}\left[-P_{T}\right]=-e^{-r \tau} \int_{-\infty}^{0} x \mathrm{~d} \psi^{\gamma}\left(F_{-P_{T}}(x)\right) \\
& =-e^{-r \tau} \int_{-\infty}^{0} x \mathrm{~d} \psi^{\gamma}\left(F_{\mathrm{GA}}(K+x)\right) \\
& =-e^{-r \tau} \int_{0}^{\infty} x \mathrm{~d} \psi^{\gamma}\left(F_{\mathrm{GA}}(K-x)\right) \\
& =e^{-r \tau} \int_{0}^{K}(K-x) \mathrm{d} \psi^{\gamma}\left(F_{\mathrm{GA}}(x)\right) \\
& =e^{-r \tau} \int_{0}^{K}(K-x) \mathrm{d} \Phi\left(\frac{\ln \left(x /\left(B_{j}^{s a} S_{t}\right)\right)-\left(r-1 / 2 \sigma^{2}\right) T_{\mu, n-j}^{s a}}{\sigma \sqrt{T_{n-j}^{s a}}}+\gamma\right) \\
& =K \Phi\left(\frac{\ln \left(K /\left(B_{j}^{s a} S_{t}\right)\right)-\left(r-1 / 2 \sigma^{2}\right) T_{\mu, n-j}^{s a}}{\sigma \sqrt{T_{n-j}^{s a}}}+\gamma\right) \\
& +B_{j}^{s a} S_{t} \exp \left(r T_{\mu, n-j}^{s a}-\frac{\sigma^{2}}{2}\left(T_{\mu, n-j}^{s a}-T_{n-j}^{s a}\right)-\gamma \sigma \sqrt{T_{n-j}^{s a}}\right) \cdot \Phi\left(\frac{\ln \left(K /\left(B_{j}^{s a} S_{t}\right)\right)-\left(r-1 / 2 \sigma^{2}\right) T_{\mu, n-j}^{s a}-\sigma^{2} T_{n-j}^{s a}}{\sigma \sqrt{T_{n-j}^{s a}}}+\gamma\right) .
\end{aligned}
$$

$$
\begin{aligned}
& b_{\gamma}(C) \cong S_{t} \bar{A}_{j}^{b} \Phi\left(\bar{d}_{n-j}^{b}+\sigma \sqrt{T_{n-j}^{s a}}\right)-e^{-r \tau} K \Phi\left(\bar{d}_{n-j}^{b}\right), \\
& a_{\gamma}(C) \cong S_{t} \bar{A}_{j}^{a} \Phi\left(\bar{d}_{n-j}^{a}+\sigma \sqrt{T_{n-j}^{s a}}\right)-e^{-r \tau} K \Phi\left(\bar{d}_{n-j}^{a}\right),
\end{aligned}
$$

Let the payoff of the European discrete arithmetic average Asian option be $C_{T}=(\mathrm{AA}(n)-K)^{+}$or $P_{T}=(K-\mathrm{AA}(n))^{+}$. By using Lemma 2 and applying the method of calculating Choquet expectation in Theorem 1, we can easily obtain the explicit approximate formulas of the discrete arithmetic average Asian options.

and the bid-ask prices of the European discrete arithmetic average Asian put option at time $t$ are given by

$$
\begin{aligned}
& b_{\gamma}(P) \cong e^{-r \tau} K \Phi\left(-\bar{d}_{n-j}^{a}\right)-S_{t} \bar{A}_{j}^{a} \Phi\left(-\bar{d}_{n-j}^{a}-\sigma \sqrt{T_{n-j}^{s a}}\right), \\
& a_{\gamma}(P) \cong e^{-r \tau} K \Phi\left(-\bar{d}_{n-j}^{b}\right)-S_{t} \bar{A}_{j}^{b} \Phi\left(-\bar{d}_{n-j}^{b}-\sigma \sqrt{T_{n-j}^{s a}}\right),
\end{aligned}
$$

Theorem 2. Assume that the distortion function $\psi^{\gamma}(u)$ is the Wang transform; then, the bid-ask prices of the European discrete arithmetic average Asian call option at time $t$ are given by

where

$$
\begin{aligned}
\bar{d}_{n-j}^{b}= & \frac{\ln \left(\left(\kappa B_{j}^{s a} S_{t}\right) / K\right)+\left(r-1 / 2 \sigma^{2}\right) T_{\mu, n-j}^{s a}}{\sigma \sqrt{T_{n-j}^{s a}}}-\gamma, \\
\bar{d}_{n-j}^{a}= & \frac{\ln \left(\left(\kappa B_{j}^{s a} S_{t}\right) / K\right)+\left(r-1 / 2 \sigma^{2}\right) T_{\mu, n-j}^{s a}}{\sigma \sqrt{T_{n-j}^{s a}}}+\gamma, \\
\bar{A}_{j}^{b} & =\kappa B_{j}^{s a} \exp \left(-r\left(\tau-T_{\mu, n-j}^{s a}\right)-\frac{\sigma^{2}}{2}\left(T_{\mu, n-j}^{s a}-T_{n-j}^{s a}\right)-\gamma \sigma \sqrt{T_{n-j}^{s a}}\right), \\
\bar{A}_{j}^{a} & =\kappa B_{j}^{s a} \exp \left(-r\left(\tau-T_{\mu, n-j}^{s a}\right)-\frac{\sigma^{2}}{2}\left(T_{\mu, n-j}^{s a}-T_{n-j}^{s a}\right)+\gamma \sigma \sqrt{T_{n-j}^{s a}}\right),
\end{aligned}
$$


TABle 1: The bid-ask prices of the European discrete geometric Asian options at different $\gamma$ with $r=0.07, \sigma=0.2, \tau=1$, $n=12, h=1 / 12$, and $j=0$.

\begin{tabular}{lcccccccc}
\hline & & & & Call & & & Put \\
& $S_{0}$ & $K$ & Ask & Bid & Spread & Ask & Bid & Spread \\
\hline \multirow{4}{*}{0} & & 90 & 13.304 & 13.304 & 0 & 0.697 & 0.697 & 0 \\
& 100 & 100 & 6.465 & 6.465 & 0 & 3.182 & 3.182 & 0 \\
& & 110 & 2.431 & 2.431 & 0 & 8.472 & 8.472 & 0 \\
\hline \multirow{4}{*}{0.03} & & 90 & 13.620 & 12.991 & 0.629 & 0.739 & 0.657 & 0.081 \\
& 100 & 100 & 6.693 & 6.242 & 0.451 & 3.313 & 3.054 & 0.260 \\
& & 110 & 2.551 & 2.315 & 0.236 & 8.710 & 8.236 & 0.474 \\
\hline \multirow{4}{*}{0.3} & & 90 & 16.601 & 10.330 & 6.272 & 1.210 & 0.376 & 0.834 \\
& 100 & 100 & 8.957 & 4.453 & 4.504 & 4.658 & 2.056 & 2.602 \\
& & 110 & 3.839 & 1.452 & 2.387 & 10.980 & 6.262 & 4.718 \\
\hline \multirow{4}{*}{0.9} & & 90 & 23.962 & 5.545 & 18.417 & 3.027 & 0.089 & 2.938 \\
& 100 & 100 & 15.264 & 1.802 & 13.462 & 8.608 & 0.715 & 7.893 \\
& & 110 & 8.172 & 0.428 & 7.744 & 16.557 & 2.947 & 13.610 \\
\hline
\end{tabular}

TABle 2: The bid-ask prices of the European discrete arithmetic Asian options at different $\gamma$ with $r=0.07, \sigma=0.2, \tau=1$, $n=12, h=1 / 12$, and $j=0$.

\begin{tabular}{ccccccccc}
\hline$\gamma$ & \multirow{2}{*}{$S_{0}$} & $K$ & & Call & & & Put \\
& & & Ask & Bid & Spread & Ask & Bid & Spread \\
\hline \multirow{4}{*}{0} & & 90 & 13.598 & 13.598 & 0 & 0.660 & 0.660 & 0 \\
& 100 & 100 & 6.676 & 6.676 & 0 & 3.063 & 3.063 & 0 \\
& & 110 & 2.543 & 2.543 & 0 & 8.253 & 8.253 & 0 \\
\hline \multirow{4}{*}{0.03} & & 90 & 13.917 & 13.282 & 0.635 & 0.700 & 0.622 & 0.078 \\
& 100 & 100 & 6.909 & 6.449 & 0.460 & 3.191 & 2.938 & 0.253 \\
& & 110 & 2.667 & 2.423 & 0.244 & 8.489 & 8.020 & 0.469 \\
\hline \multirow{4}{*}{0.3} & & 90 & 16.922 & 10.591 & 6.331 & 1.153 & 0.354 & 0.799 \\
& 100 & 100 & 9.213 & 4.620 & 4.592 & 4.506 & 1.968 & 2.537 \\
& & 110 & 3.994 & 1.527 & 2.467 & 10.736 & 6.074 & 4.663 \\
\hline \multirow{4}{*}{0.9} & & 90 & 24.325 & 5.730 & 18.595 & 2.915 & 0.082 & 2.833 \\
& 100 & 100 & 15.595 & 1.889 & 13.706 & 8.399 & 0.676 & 7.722 \\
& & 110 & 8.425 & 0.455 & 7.969 & 16.289 & 2.830 & 13.459 \\
\hline
\end{tabular}

and $B_{j}^{s a}, T_{n-j}^{s a}, T_{\mu, n-j}^{s a}$, and $\kappa$ are the same as in Lemmas 1 and 2. In particular, for $\gamma=0$, the bid-ask prices are equivalent, and the bid-ask prices of the European discrete arithmetic average Asian option reduce to the B-S-type formulas in [1].

Remark 1. If the observation frequency $h$ is continuous, the number of observations $n$ approaches to infinity, and the observation frequency $h$ tends to zero. Substituting $n \longrightarrow \infty$ and $h \longrightarrow 0,(n-1) h \longrightarrow \tau$, into $T_{n-j}^{s a}$ and $T_{\mu, n-j}^{s a}$ yields $T_{n-j}^{s a} \longrightarrow \tau / 2$ and $T_{\mu, n-j}^{s a} \longrightarrow \tau / 3$. Furthermore, substituting these two limiting values into (25) and (27), we obtain the bid-ask prices of a continuous geometric average Asian option in [18]. On the contrary, substituting these two limiting values into (42) and (43), we can also derive the bidask prices of a continuous arithmetic average Asian option.

\section{Numerical Examples}

In this section, we give two examples to demonstrate and validate that the approximate closed forms are efficient and accurate. Suppose there are 12 observations in the European discrete geometric average or arithmetic average Asian option, observation frequency in monthly, the averaging period has not started, and the time to maturity of the option is one year. That is to say, $n=12, h=1 / 12, j=0$, and $\tau=1$. In addition, assume that the risk-free interest rate $r=0.07$, initial stock price $S_{0}=100$, and the stock price volatility $\sigma=0.2$. By substituting these parameters into the approximate closed forms with the different market liquidity parameter $\gamma$ and strike price $K$, we will obtain the numerical results which are displayed in Tables 1 and 2.

In Table 1, we provide the numerical results for the bidask prices of the discrete geometric average Asian options. And Table 2 shows the numerical results for the bid-ask prices of the discrete arithmetic average Asian options. For $\gamma=0$, the ask and bid prices are equivalent, and they reduce to the B-S-type formulas presented by Zhang [1]. From Tables 1 and 2, we find that the higher the liquidity level $\gamma$, the wider the bid-ask spread, which means that liquidity is insufficient.

\section{Conclusion}

In this paper, within the framework of conic finance, we derive effective, explicit, approximate formulas to estimate the bid-ask prices for the European discrete geometric average and arithmetic average Asian options. Finally, numerical examples show that the theory of conic finance has significant contributions to financial derivative pricing.

\section{Data Availability}

The numerical simulation data used to support the findings of this study are included within the article.

\section{Conflicts of Interest}

The authors declare that there are no conflicts of interest regarding the publication of this paper.

\section{Acknowledgments}

This work was supported by the Foundation of Yibin University, China (Grant no. 2019QD07).

\section{References}

[1] P. G. Zhang, Exotic Options:A Guide to Second Generation Options, World Scientific Publishing Co. Pte. Ltd, Singapore, 2nd edition, 1998.

[2] J. C. Hull, Options, Futures, and Other Derivatives, PrenticeHall, Hoboken, NJ, USA, 7th edition, 2009.

[3] P. Carr, H. Geman, D. B. Madan, and M. Yor, "Stochastic volatility for lévy processes," Mathematical Finance, vol. 13, no. 3, pp. 345-382, 2003.

[4] J. Han and J. Arnulf, "Deep learning-based numerical methods for high-dimensional parabolic partial differential equations and backward stochastic differential equations," Communications in Mathematics \& Statistics, vol. 5, pp. 349-380, 2017. 
[5] C.-F. Ivaşcu, "Option pricing using machine learning," Expert Systems with Applications, vol. 163, pp. 113799-113806, 2021.

[6] J. H. Jang, J. Yoon, J. Kim, J. Gu, and H. Y. Kim, "Deepoption: a novel option pricing framework based on deep learning with fused distilled data from multiple parametric methods," Information Fusion, vol. 70, pp. 43-59, 2021.

[7] D. B. Madan and A. Cherny, "Markets as a counterparty: an introduction to conic finance," International Journal of Theoretical and Applied Finance, vol. 13, no. 8, pp. 1149-1177, 2010.

[8] A. Cherny and D. Madan, "New measures for performance evaluation," Review of Financial Studies, vol. 22, no. 7, pp. 2571-2606, 2009.

[9] D. Madan, "Conserving capital by adjusting deltas for gamma in the presence of skewness," Journal of Risk and Financial Management, vol. 3, no. 1, pp. 1-25, 2010.

[10] M. Leippold and S. Schärer, "Discrete-time option pricing with stochastic liquidity," Journal of Banking \& Finance, vol. 75, pp. 1-16, 2017.

[11] M. Leippold and S. Schaerer, "Optimal conic execution strategies with stochastic liquidity," in SSRN Electronic Journal, pp. 1-44, Social Science Electronic Publishing, Rochester, NY, USA, 2018.

[12] D. B. Madan and W. Schoutens, "Conic option pricing," The Journal of Derivatives, vol. 25, no. 1, pp. 10-36, 2017.

[13] F. Guillaume, G. Junike, P. Leoni, and W. Schoutens, "Implied liquidity risk premia in option markets," Annals of Finance, vol. 15, no. 2, pp. 233-246, 2019.

[14] H. Albrecher, F. Guillaume, and W. Schoutens, "Implied liquidity: model sensitivity," Journal of Empirical Finance, vol. 23, pp. 48-67, 2013.

[15] F. Guillaume, "The lix: a model-independent liquidity index," Journal of Banking \& Finance, vol. 58, pp. 214-231, 2015.

[16] D. Madan and W. Schoutens, Applied Conic Finance: Conic Finance, Cambridge University Press, Cambridge, UK, 2016.

[17] M. Energy Sonono and H. Phillip Mashele, "Estimation of bid-ask prices for options on libor based instruments," Finance Research Letters, vol. 19, pp. 33-41, 2016.

[18] T. Chen, K. Xiang, and X. Luo, "Estimation of ask and bid prices for geometric asian options," Discrete Dynamics in Nature and Society, vol. 2019, pp. 1-9, 2019.

[19] G. Choquet, "Theory of capacities," Annales de l'institut Fourier, vol. 5, pp. 131-295, 1954.

[20] S. S. Wang, "A class of distortion operators for pricing financial and insurance risks," The Journal of Risk and Insurance, vol. 67, no. 1, pp. 15-36, 2000.

[21] S. S. Wang, "A universal framework for pricing financial and insurance risks," Astin Bulletin, vol. 32, no. 2, pp. 213-234, 2002.

[22] A. G. Z. Kemna and A. C. F. Vorst, "A pricing method for options based on average asset values," Journal of Banking \& Finance, vol. 14, no. 1, pp. 113-129, 1990.

[23] S. M. Turnbull and L. M. Wakeman, "A quick algorithm for pricing european average options," The Journal of Financial and Quantitative Analysis, vol. 26, no. 3, pp. 377-420, 1991. 